\title{
Haciendo nada: reflexiones en torno a las políticas de desempleo juvenil
}

\author{
Maricela González Moya*
}

\begin{abstract}
RESUMEN
El artículo reflexiona en torno al tema del desempleo juvenil y repasa algunas políticas para enfrentar el problema. Se afirma que, pese a las medidas tomadas, en muchos países el desempleo de los jóvenes ha seguido en aumento y se examinan las hipótesis que lo explican. Asimismo, se proponen algunas líneas como el apoyo a la inserción al primer empleo, a la empleabilidad, al emprendimiento y a grupos específicos especialmente vulnerables como es el caso de mujeres, discapacitados y desertores del sistema escolar. Finalmente, se rescatan experiencias internacionales exitosas y se señalan desafíos para abordar el desempleo juvenil en Chile.
\end{abstract}

Palabras clave: Desempleo juvenil / Políticas de empleo juvenil / explicaciones del desempleo juvenil

\section{Doing nothing at all: reflections regarding the policies of youth unemployment}

\begin{abstract}
This article makes a reflection regarding the issue of youth unemployment and reviews some policies to put up with the problem. Some people state that, in spite of the measures that have been taken, in many countries, young people's unemployment rate has continued to increase, and the hypotheses that explain this result are examined herein. In addition, some guidelines are proposed, such as the support given for the first employment, for the employability, for the venturing and for specific groups, especially those most vulnerable, as it is the case of women, the disabled and school dropouts. Finally, successful international experiences are shared and challenges are pointed out to deal with youth unemployment in Chile.
\end{abstract}

Key words: youth unemployment / youth employment policies / explanations for youth unemployment

* Chilena. Trabajadora Social, Magíster en Filosofía Política USACH. Directora Escuela de Trabajo Social, Universidad Santo Tomás.

Correo electrónico:mgonzalez@santotomas.cl 


\section{El desempleo juvenil}

Uno de los problemas más graves del mercado de trabajo es el de la inserción de los jóvenes a él. Éste refleja el nivel de correspondencia entre la oferta y la demanda de trabajo en una sociedad determinada, a su vez, el nivel en que esta sociedad aprovecha su capacidad productiva y el ritmo de desarrollo al que avanza.

Al mismo tiempo, el desempleo juvenil y la precariedad de la condición laboral de los jóvenes no sólo repercuten negativamente en el crecimiento económico y en la calidad de vida general de la población de un país, sino que, como lo señalan García y Gutiérrez, el desempleo juvenil es además, "un magnífico observatorio de los procesos de generación de desigualdad económica" (García y Gutiérrez 1996: 270). En primer lugar, porque es posible observar en los jóvenes la incidencia en la vida laboral de los factores educacionales y familiares; segundo, porque en esta primera fase suele notarse también la lógica de la movilidad económica; por último, porque resultan más evidentes allí la desigualdad de acceso y al interior del mercado de trabajo" (García y Gutiérrez 1996: 270)

Una inserción laboral defectuosa o débil significa, además, un mal uso del capital humano, una ineficiencia en las inversiones en educación y capacitación, una propensión a la transmisión intergeneracional de la pobreza, una prolongación de los vínculos de dependencia con respecto al hogar de origen y un paso decidido hacia la conformación de un grupo social con mayores problemas de adaptación y marginación social (Weller 2003: 10)

En América Latina el problema es preocupación pública y se ha visto agravado por la expectativa que existió respecto de un hipotético mejoramiento en los indicadores de desocupación juvenil. Se había depositado confianza en que el descenso demográfico, junto con la evolución de los sistemas educativos, iban a generar una disminución de la presión por trabajar y un mejoramiento de la empleabilidad y de la calidad de la mano de obra (Weller 2203: 11). Contrariamente, el desempleo juvenil ha crecido. Mientras que la tasa de participación de la fuerza laboral juvenil está disminuyendo a nivel mundial (cuadro $\mathrm{N}^{\circ} 1$ ), el crecimiento de la población juvenil sigue superando el crecimiento del empleo (cuadro $\mathrm{N}^{\circ} 2$ ) y esa diferencia no se explica exclusivamente por la mayor permanencia de los jóvenes en el sistema educativo. 
Cuadro $\mathrm{N}^{\circ}$ 1. Tasa de participación juvenil en la fuerza de trabajo por género, 1995 y 2005

\begin{tabular}{|l|c|c|c|c|c|c|}
\hline \multirow{2}{*}{} & \multicolumn{2}{|c|}{ TOTAL } & \multicolumn{2}{l|}{ HOMBRES } & \multicolumn{2}{c|}{ MUJERES } \\
\cline { 2 - 7 } & 1995 & 2005 & 1995 & 2005 & 1995 & 2005 \\
\hline Mundo & 58,9 & 54,7 & 67,2 & 63,0 & 50,1 & 45,9 \\
\hline Economías Desarrolladas y Unión Europea & 53,6 & 51,8 & 56,9 & 54,0 & 50,1 & 49,6 \\
\hline Europa Central y Oriental & 47,2 & 41,8 & 52,8 & 48,4 & 41,5 & 35,0 \\
\hline Asia Oriental & 75,2 & 67,3 & 74,6 & 66,3 & 75,7 & 68,4 \\
\hline Sudeste de Asia y Pacífico & 58,1 & 56,5 & 64,5 & 64,2 & 51,6 & 48,5 \\
\hline Asia del Sur & 50,6 & 47,2 & 68,0 & 64,2 & 31,7 & 29,1 \\
\hline América Latina y el Caribe & 56,4 & 54,2 & 70,4 & 63,8 & 42,3 & 44,5 \\
\hline Oriente Medio y África del Norte & 40,0 & 40,0 & 56,2 & 54,3 & 23,2 & 25,1 \\
\hline África subsahariana & 68,2 & 65,5 & 76,1 & 73,7 & 60,2 & 57,3 \\
\hline
\end{tabular}

Fuente: OIT (2006) Modelo de Tendencias Mundiales de Empleo. Citado en: OIT, Tendencias Mundiales del Empleo Juvenil. Ginebra. Pág. 16

Cuadro $\mathrm{N}^{\circ}$ 2. Relación empleo-población juvenil

\begin{tabular}{|l|c|c|c|}
\hline \multirow{2}{*}{} & \multicolumn{3}{|c|}{ Relación empleo-población juvenil } \\
\cline { 2 - 4 } & 1995 & 2005 & Cambio \% 1995-2005 \\
\hline Mundo & 51,6 & 47,3 & $-8,3$ \\
\hline Economías Desarrolladas y Unión Europea & 45,4 & 45 & $-0,9$ \\
\hline Europa Central y Oriental & 38,0 & 33,5 & $-11,8$ \\
\hline Asia Oriental & 69,5 & 62,1 & $-10,6$ \\
\hline Sudeste de Asia y Pacífico & 52,8 & 47,5 & $-10,0$ \\
\hline Asia del Sur & 45,6 & 42,5 & $-6,8$ \\
\hline América Latina y el Caribe & 48,3 & 45,2 & $-6,4$ \\
\hline Oriente Medio y África del Norte & 28,5 & 29,7 & 4,2 \\
\hline África subsahariana & 56,2 & 53,7 & $-4,4$ \\
\hline
\end{tabular}

Fuente: OIT (2006) Modelo de Tendencias Mundiales de Empleo. Citado en: OIT, Tendencias Mundiales del Empleo Juvenil. Ginebra. Pág. 17

Concretamente, el número de jóvenes desempleados aumentó en 14,8\% entre 1995 y 2005. Como lo muestra el Cuadro №3, sólo descendió significativamente en las economías desarrolladas y los países de la Unión Europea, pero en todas las demás regiones aumentó y en América Latina lo hizo en un $23 \%$.

Cuadro № 3. Desempleo juvenil total, 1995, 2004 y 2005

\begin{tabular}{|l|r|r|r|c|}
\hline \multirow{2}{*}{} & \multicolumn{4}{|c|}{ Desempleo Juvenil ('000s) } \\
\cline { 2 - 5 } & 1995 & 2004 & 2005 & $\begin{array}{l}\text { Cambio \% } \\
1995-2005\end{array}$ \\
\hline Mundo & 74.302 & 84.546 & 85.278 & 14,8 \\
\hline $\begin{array}{l}\text { Economías Desarrolladas y Unión } \\
\text { Europea }\end{array}$ & 10.281 & 8.997 & 8.481 & $-17,5$ \\
\hline Europa Central y Oriental & 5.962 & 5.724 & 5.900 & $-1,0$ \\
\hline Asia Oriental & 13.149 & 11.840 & 12.076 & $-8,2$ \\
\hline
\end{tabular}




\begin{tabular}{|l|l|l|l|l|}
\hline Sudeste de Asia y Pacífico & 5.242 & 9.687 & 9.727 & 85,5 \\
\hline Asia del Sur & 11.765 & 13.561 & 13.662 & 16,1 \\
\hline América Latina y el Caribe & 7.722 & 9.263 & 9.495 & 23,0 \\
\hline Oriente Medio y África del Norte & 7.209 & 8.380 & 8.525 & 18,2 \\
\hline África subsahariana & 12.972 & 17.095 & 17.414 & 34,2 \\
\hline
\end{tabular}

Fuente: OIT (2006) Modelo de Tendencias Mundiales de Empleo. Citado en: OIT, Tendencias Mundiales del Empleo Juvenil. Ginebra. Pág. 18

Como lo muestra el Cuadro $N^{\circ} 4$, también aumentó la tasa de desempleo mundial juvenil.

Cuadro $\mathrm{N}^{\circ} 4$. Tasas de desempleo mundial juvenil y de desempleo juvenil 1995-2005

\begin{tabular}{|l|c|c|c|c|c|c|c|c|c|c|c|}
\hline & $\mathbf{1 9 9 5}$ & $\mathbf{1 9 9 6}$ & $\mathbf{1 9 9 7}$ & $\mathbf{1 9 9 8}$ & $\mathbf{1 9 9 9}$ & $\mathbf{2 0 0 0}$ & $\mathbf{2 0 0 1}$ & $\mathbf{2 0 0 2}$ & $\mathbf{2 0 0 3}$ & $\mathbf{2 0 0 4}$ & $\mathbf{2 0 0 5}$ \\
\hline $\begin{array}{l}\text { Desempleo } \\
\text { juvenil } \\
\text { (millones) }\end{array}$ & 74,3 & 74,4 & 75,7 & 77,3 & 79,3 & 79,9 & 82,7 & 84,4 & 83,3 & 84,5 & 85,3 \\
\hline $\begin{array}{l}\text { Tasa de } \\
\text { desempleo } \\
\text { juvenil (\%) }\end{array}$ & 12,3 & 12,4 & 12,7 & 13,0 & 13,2 & 13,3 & 13,7 & 13,8 & 13,6 & 13,5 & 13,5 \\
\hline
\end{tabular}

Fuente: OIT (2006) Modelo de Tendencias Mundiales de Empleo. Citado en: OIT, Tendencias Mundiales del Empleo Juvenil. Ginebra. Pág. 18

América Latina se encuentra entre las regiones que presenta mayores índices de desempleo juvenil, tanto en su globalidad como en la mayor parte de los países que la conforman. Como se aprecia en los cuadros $\mathrm{N}^{\circ} 5$ y N $\mathrm{N}^{\circ} 6$, el desempleo juvenil creció en países como Argentina, Bolivia, Chile, Costa Rica, Ecuador, Honduras, Perú, Santa Lucía y Uruguay, y en casi todos aumentó también la distancia entre el desempleo total y el desempleo juvenil (Gráfico No 1 ).

Cuadro $N^{\circ} 5$. Tasas de desempleo juvenil en América Latina por sexo 19952004

\begin{tabular}{|l|c|c|c|c|c|c|c|c|c|c|c|c|c|c|c|c|c|c|c|}
\hline & \multicolumn{9}{|c|}{ Hombres } & \multicolumn{6}{|c|}{ Ambos Sexos } \\
\hline & 1995 & 2000 & 2001 & 2002 & 2003 & 2004 & 1995 & 2000 & 2001 & 2002 & 2003 & 2004 & 1995 & 2000 & 2001 & 2002 & 2003 & 2004 \\
\hline Argentina & & 31,6 & & & 34,3 & & & 26,2 & & & 33,7 & & & 28,5 & & & 33,9 & \\
\hline Bahamas & 24 & & 16,6 & 21,9 & 28,2 & 26,9 & 18,5 & & 14,0 & 17,9 & 20,3 & 20,0 & 21,0 & & 15,1 & 19,9 & 24,0 & 23,3 \\
\hline Barbados & 43,2 & 21,8 & 26,0 & 26,0 & & & 33,3 & 17,6 & 20,7 & 21,3 & & & 37,8 & 19,4 & 23,1 & 23,4 & & \\
\hline Bolivia & 7,0 & 10,4 & & & & & 5,2 & 7,0 & & & & & 6,1 & 8,5 & & & & \\
\hline Brasil & 14,1 & & 22,4 & & & & 9,7 & & 14,6 & & & & 11,4 & & 17,9 & & & \\
\hline Chile & 12,7 & 24,0 & 22,1 & 22,4 & 22,5 & & 10,8 & 17,6 & 17,1 & 18,8 & 17,1 & & 11,5 & 19,8 & 18,8 & 20,2 & 19,0 & \\
\hline Costa Rica & 12,9 & 14,5 & 16,4 & & 18 & & 10,2 & 9,1 & 11,9 & & 12,9 & & 11,1 & 11,0 & 13,4 & & 14,7 & \\
\hline Ecuador & 18,6 & 18,1 & 20,4 & 25,5 & 24,9 & & 13,0 & 10,5 & 10,6 & 12,0 & 19,3 & & 15,3 & 13,5 & 14,8 & 17,4 & 21,6 & \\
\hline $\begin{array}{l}\text { El } \\
\text { Salvador }\end{array}$ & 13,0 & & & 8,4 & 7,7 & & 13,4 & & & 13,1 & 13,5 & & 13,3 & & & 11,5 & 11,4 & \\
\hline Honduras & 6,6 & & & & 12,1 & & 4,6 & & & & 6,1 & & 5,2 & & 7,2 & 6,0 & 7,9 & \\
\hline
\end{tabular}




\begin{tabular}{|l|c|c|c|c|c|c|c|c|c|c|c|c|c|c|c|c|c|c|}
\hline Jamaica & 44,6 & 42,8 & 44,1 & 39,6 & 32,2 & & 25,1 & 23,8 & 24,6 & 23,6 & 22,1 & & 34,1 & 32,1 & 33,0 & 30,8 & 26,4 & \\
\hline México & 11,0 & 4,7 & 5,0 & 5,6 & 6,2 & 7,8 & 8,9 & 4,2 & 3,6 & 4,5 & 4,9 & 5,6 & 9,6 & 4,4 & 4,1 & 4,9 & 5,3 & 6,4 \\
\hline Nicaragua & 24,7 & 13,6 & 19,7 & & 15,8 & & 22,4 & 7,0 & 20,3 & & 10,8 & & 23,3 & 9,1 & 20,0 & & 12,5 & \\
\hline Panamá & 35,6 & 36,6 & & 38,9 & 38,3 & & 23,0 & 24,7 & & 24,4 & 24,2 & & 27,3 & 29,0 & & 29,3 & 292 & \\
\hline Paraguay & 7,9 & & 17,3 & & & & 5,2 & & 11,7 & & & & 6,0 & & 13,8 & & & \\
\hline Perú & & 13,6 & 13,9 & & 20,7 & & & 13,7 & 12,6 & & 17,6 & & & 13,6 & 13,2 & & 19,2 & \\
\hline $\begin{array}{l}\text { Santa } \\
\text { Lucía }\end{array}$ & 42,3 & 40,5 & & 41,2 & 49,2 & & 27,5 & 32,2 & & 31,7 & 31,8 & & 34,2 & 36,1 & & 39,9 & 40,0 & \\
\hline $\begin{array}{l}\text { Trinidad y } \\
\text { Tobago }\end{array}$ & 35,2 & 27,6 & & 26,4 & & & 28,3 & 20,3 & & 17,4 & & & 31,0 & 23,2 & & 21,1 & & \\
\hline Uruguay & 29,2 & 36,4 & 41,7 & & 44,2 & & 21,3 & 26,6 & 28,7 & & 34,1 & & 24,6 & 30,7 & 34,2 & & 38,3 & \\
\hline Venezuela & & 28,3 & 27,7 & 34,5 & 34,8 & & & 22,3 & 19,6 & 24,4 & 23,7 & & & 24,3 & 22,6 & 28,2 & 28,0 & \\
\hline
\end{tabular}

Fuente: ECLAC (2006) Statistical Yearbook for Latin America and the Caribean. Pág. 56

Gráfico $\mathrm{N}^{\circ} 1$. Tasas de desempleo juvenil y desempleo global en América Latina 1993

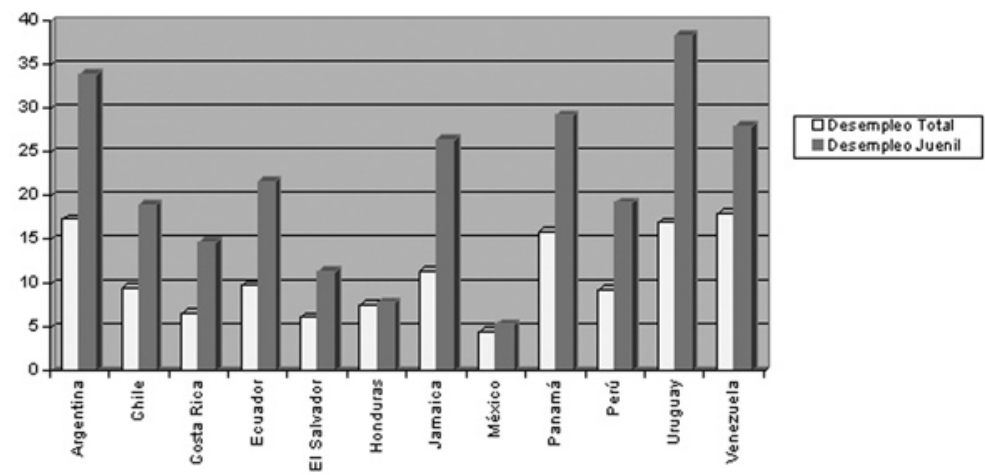

Fuente: ECLAC (2006) Statistical Yearbook for Latin America and the Caribean. Págs. $54-56$

Como lo muestra María Antonia Gallart (Gallart 2003: 7-8), la inserción laboral de los jóvenes está también condicionada por factores socioeconómicos, de edad y de género. La tendencia es a que los jóvenes entre 15 y 19 años sigan estudiando y posterguen su entrada al mercado de trabajo, mientras que los de 20 a 24 años aumenten su participación laboral. Pese a ello, la cesantía es mayor en inversa proporcionalidad a la edad, como lo muestra el Gráfico № 2: 
Gráfico № 2. América latina (16 países). Tasa de Desempleo por tramos etéreos, áreas urbanas, 1990-2002

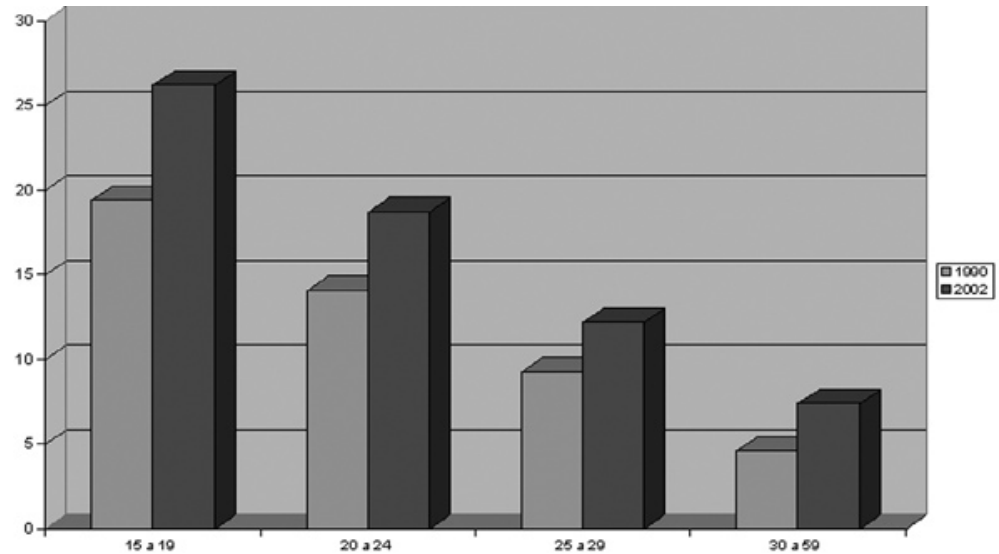

Fuente: CEPAL. Citado en: Schkolnick, Mariana (2005) Caracterización de la Inserción Laboral de los Jóvenes. Pág. 31

El desempleo juvenil es mayor en los sectores de menores ingresos. Los jóvenes de los dos quintiles inferiores tuvieron un leve crecimiento en sus tasas de actividad (de 53,3 a 54,8\%), pero las de los dos quintiles inferiores descendieron (de 62 a $60 \%$ ). El desempleo de los jóvenes pobres tiende a ser casi tres veces superior al de los jóvenes no pobres en algunos países de América Latina (Cuadro $\mathrm{N}^{\circ} 6$ ):

Cuadro $\mathrm{N}^{\circ} 6$. Tasas de desempleo juvenil en cuatro países de América Latina, en porcentaje, año 1997, en jóvenes pobres y no pobres

\begin{tabular}{|l|c|c|c|c|c|c|c|c|}
\hline & ARGENTINA & \multicolumn{2}{|c|}{ CHILE } & \multicolumn{2}{c|}{ COLOMBIA } & \multicolumn{2}{c|}{ PERÚ } \\
\hline & Pobres & $\begin{array}{c}\text { No } \\
\text { Pobres }\end{array}$ & Pobres & $\begin{array}{c}\text { No } \\
\text { Pobres }\end{array}$ & Pobres & $\begin{array}{c}\text { No } \\
\text { Pobres }\end{array}$ & Pobres & $\begin{array}{c}\text { No } \\
\text { Pobres }\end{array}$ \\
\hline $\begin{array}{l}\text { Tasa de } \\
\text { Desempleo }\end{array}$ & 46,6 & 18,4 & 8,4 & 3,6 & 35,2 & 19,8 & 19,3 & 10,3 \\
\hline
\end{tabular}

Fuente: CEPAL (2003) Encuestas de Hogares, Argentina, Chile, Colombia y Perú. Extraído de: Gallart, María Antonia: La formación para el trabajo y los jóvenes en América Latina. CEPAL. Pág. 17

Con respecto a la tasa de desocupación femenina, se evidencia que las cifras casi se triplicaron (Cuadro $N^{\circ}$ 5). Por último, en relación con la educación, han mejorado los niveles de instrucción de los jóvenes, pero han también aumentado las dificultades para conseguir empleo: “... al principio de la década el 30\% de los jóvenes desocupados había alcanzado menos de seis años de estudio; al fin de ellas sólo el 21\% estaba en ese nivel. Por otro lado las diferencias de ingresos entre los jóvenes trabajadores que habían realizado estudios 
terciarios y los demás se habían incrementado. El ingreso medio de los trabajadores que habían superado la educación secundaria (13 años y más) era 4,6 veces mayor que el de los que sólo registraban cinco años de escolaridad o menos" (Gallart 2003: 8).

\section{Hipótesis explicativas acerca del desempleo juvenil}

Existen varias hipótesis que intentan explicar las causas del desempleo juvenil y, sobre todo, del hecho de que éste sea siempre mayor que las tasas generales de desempleo en un mismo lugar. Partiremos por las hipótesis más generales, para desplazarnos a algunos aspectos específicos posteriormente.

Una primera hipótesis sostiene que el desempleo juvenil está estrechamente vinculado con el crecimiento económico y la demanda agregada general de un país. Frente a condiciones económicas adversas, los jóvenes resienten más el desempleo pues carecen de formación específica y de la antigüedad que sí tienen los trabajadores adultos (OIT 2005: 9-10); a su vez, en momentos de reactivación económica, las empresas suelen contratar primero a los desempleados con más experiencia laboral. "En consecuencia, las difíciles perspectivas de inserción laboral en términos de la probabilidad de conseguir un empleo y ganar un ingreso que corresponde a las expectativas lleva a un elevado número de jóvenes a desertar del sistema educativo, sin insertarse al mercado de trabajo, lo que en muchos casos los condena a la marginalidad [...] De esta manera, la inserción laboral productiva de los y las jóvenes requeriría de un periodo relativamente prolongado de crecimiento estable..." (Weller 2003: 17)

Además de esto, está el argumento del "trabajador adicional". En síntesis, éste sostiene que cuando se deterioran los ingresos labo-

1 Es interesante complementar lo anterior con lo que señala Mariana Schkolnik, al sostener que "en periodos de crisis económicas los jóvenes se desincentivan de ingresar al mercado de trabajo y continúan estudios. A la inversa, en periodos de bonanza abandonan los estudios y se interesan más por trabajar, ante la promesa de mejores ingresos. Este comportamiento anti-cíclico, sin embargo, no se aplica a los jóvenes de los estratos socioeconómicos más bajos; para ellos sigue siendo válida la relación crisis económica-desempleo juvenil, pues ante la cesantía de sus padres, a quienes azota más profundamente, deben buscar imperativamente un empleo" (Schkolnik; 2005: 13). 
rales de las familias, aumenta la oferta laboral y, por ende, los jóvenes pasan a constituirse en fuerza de trabajo secundaria (sobre todo después de la incorporación creciente de mujeres adultas al trabajo), tienden a abandonar los estudios y, a la larga, a convertirse en desempleados crónicos o trabajadores precarios.

La volatilidad económica, además, hace que la incertidumbre del mercado de bienes se traspase al mercado de trabajo. En contextos de este tipo, las empresas cuidan más a sus trabajadores, en especial a los que tienen mayores calificaciones o capital humano específico y tienden a no contratar personal nuevo o sin experiencia laboral, en un intento por reducir la incerteza.

En segundo lugar, tenemos las hipótesis referidas a la incongruencia entre la demanda y la oferta de trabajo juvenil. En este caso lo que se afirma es que los jóvenes están mal preparados para dar respuesta a las demandas del aparato productivo, sobre todo en las condiciones actuales, en las cuales esas demandas suelen cambiar rápidamente, debido a la aceleración de las transformaciones tecnológicas ${ }^{2}$.

Los programas de capacitación y educación resultan ineficaces por dos razones: en primer lugar porque están referidos a la demanda, pero ésta varía de acuerdo a una dinámica difícil de seguir. Algunas instituciones educativas, especialmente las menos ágiles, no son capaces de ajustar sus programas a este respecto y los requerimientos mismos van variando a una velocidad cada vez mayor y ni siquiera los potenciales empleadores logran tener certeza sobre el futuro de éstos.

Por otro lado, los ajustes en educación impactan en el mercado de trabajo sólo en el largo plazo. "Esto es así, primero, porque toma tiempo hasta que los alumnos capacitados según nuevas orientaciones, más aptas para satisfacer la demanda, entran al mercado de trabajo. Segundo, toma tiempo hasta que el mercado de trabajo haya reconocido los avances correspondientes, dado que en un ini-

2 “...hoy en día las características de la demanda sufren continuos cambios, sobre todo en tiempos de aceleradas transformaciones tecnológicas, y los programas de educación y capacitación requieren ajustes frecuentes. Además, lo requerido son cada vez más habilidades como creatividad, capacidad de aprender continua e independientemente, manejo de información, etc., en vez del manejo repetido y poco variado de instrumentos y procesos" (Weller 2003: 14). 
cio sólo el mismo proceso productivo puede verificar lo adecuado de los programas de educación y capacitación, y sólo posteriormente los certificados correspondientes ayudan a mejorar el flujo de información" (Weller; 2003: 14).

Como señala Weller (2003), una variante de esta hipótesis señala que la expansión de la educación en las sociedades modernas tardías ocurrió de manera inadecuada, lo que generó, entre otras cosas, el fenómeno de la sobrecalificación. En primer lugar, esto ha implicado que las personas, dotadas de mejores cualificaciones, no pueden acceder a puestos de trabajo porque sus habilidades y conocimientos exceden los requeridos. En segundo lugar, ocurriría también un fenómeno opuesto: debido a que no han pasado por ninguna transformación tecnológica y que, por ende, no han cambiado sus requerimientos, en la práctica sí los aumentan porque el nivel educativo general de la población, y de quienes aspiran específicamente a esos puestos, ha subido. "Esto implica que personas cuya calificación 'objetivamente' satisface los requisitos relacionados con un empleo específico al cual previamente habría podido acceder, ahora se ven desplazadas por personal de mayor nivel educativo. En este sentido un mayor nivel educativo observado en ciertas ocupaciones no refleja una demanda por calificaciones más altas" (Weller 2003: 15).

Finalmente, también se ha señalado con respecto a la incongruencia demanda-oferta, que más que incidir el nivel educativo, lo que sí importa es el grado de experiencia del trabajador. Contrariamente a lo que se pensaba con respecto a que el cambio tecnológico favorecería a aquéllos que dispusieran de los nuevos conocimientos y habilidades requeridas, en la práctica es la combinación entre manejo tecnológico y experiencia laboral la que favorece una inserción laboral exitosa y, en esos términos, los jóvenes entran en desventaja. Como señala Schkolnik, "El incremento de la competitividad de las empresas induce a que muchas minimicen sus riesgos contratando sólo trabajadores que han tenido experiencia práctica en el rubro. Lo anterior implica una permanente discriminación negativa en contra de los jóvenes, aun cuando tengan más alta escolaridad que los adultos. Ya que su 'saber hacer' está aún poco desarrollado. Ni siquiera el diferencial de salarios entre jóvenes y adultos permite compensar desde el punto de vista del empresario, la falta de experiencia, ya sea porque no tienen tiempo para capacitarlos en la propia empresa, o bien porque no existe una adecuada oferta de 
centros e instituciones de capacitación laboral en la mayor parte de los países de América Latina" (Schkolnik 2005: 14).

Es habitual también en nuestro continente la existencia de un mercado de trabajo más rígido en el sector formal que en el caso de Europa, por ejemplo; esa rigidez afecta la inserción laboral de los jóvenes a lo menos en dos sentidos: el sector formal demanda niveles mínimos de escolaridad secundaria (más que por razones de posesión de conocimientos o destrezas, como certificado de comportamiento y disciplina), lo que castiga fuertemente a los desertores del sistema educativo; al no existir una certificación de competencias, sólo el sector informal termina por absorber este tipo de oferta. En segundo lugar, el mercado formal dispone también de menos posibilidades de contratación por jornadas parciales o sistemas de parttime u otros, que en el caso de los países europeos constituye una importante fuente para adquirir las primeras certificaciones y experiencias laborales (Schkolnik 2005: 14).

En tercer lugar, tenemos a las hipótesis sobre el funcionamiento del mercado de trabajo con prescindencia de otras variables. En este sentido, un factor que actuaría de forma relevante serían las aspiraciones incongruentes de los jóvenes con respecto a su proceso de inserción laboral. En ese sentido, mientras los jóvenes permanecen cobijados al alero de la familia de origen, pueden permanecer más tiempo buscando trabajo, hasta que encuentren uno que se adapte mejor a sus expectativas. Como consecuencia de esto, los jóvenes tienen periodos más largos de búsqueda y tasas de desempleo más altas que las de los adultos.

Además de lo anterior, el funcionamiento del mercado del trabajo con información incompleta se acentúa aún más en el caso de los jóvenes, pues ellos tienen menos datos de las empresas, de los empleadores y de las condiciones generales de trabajo y, a su vez, las empresas también tienen menos información sobre ellos, debido a su carencia de experiencia y datos laborales. Esta falta de información de ambas partes condiciona la permanencia menor de los jóvenes en los primeros puestos de trabajo, puesto que cualquiera de las partes puede no quedar satisfecha por desconocimiento.

En conjunto, estos dos argumentos llevarían a algunos autores a sostener que el desempleo juvenil, en realidad es una especie de "ilusión óptica", más que un problema propiamente tal. Como afir- 
ma Eduardo Martínez "los datos sugieren que la alta tasa de desocupación juvenil es un fenómeno natural -una especie de 'ilusión óptica'-que resulta del proceso de entrada de los jóvenes al mercado de trabajo y la demora normal en insertarse en el empleo. Y al fenómeno señalado hay que agregar el hecho -también normal- de que en los periodos de recesión o ajuste económico los jóvenes tienden a perder sus empleos antes que los adultos. Cabe entonces concluir que, en general, los jóvenes no parecen estar enfrentando mayores dificultades que los adultos cesantes en la búsqueda de trabajo, ya que la duración promedio del desempleo en ambos grupos es cercano a los tres meses, por lo menos en periodos de actividad económica plena. Ciertamente, esta conclusión no significa negar la importancia del problema de la desocupación de los jóvenes, sino simplemente señalar que el hecho de que la tasa de desocupación juvenil duplique la de los adultos es un fenómeno estructural, propio de la dinámica del mercado de trabajo" (Martínez; 1997 101).

La legislación laboral introduciría también algunas distorsiones al mercado que perjudicarían a los jóvenes a la hora de encontrar empleo, específicamente a nivel de salario mínimo. En concreto, sostiene esta hipótesis es que la política del salario mínimo, al no diferenciar entre distintos niveles de experiencia y, por ende, de productividad, genera que las empresas contraten a personas adultas. Por otro lado, dicho salario, cuando sobrepasa el de reserva de los jóvenes, redunda en mayor desempleo, pues constituye un incentivo para la deserción escolar. Otras leyes laborales, como las destinadas a proteger la estabilidad en el trabajo, perjudican también a los jóvenes, pues los empleadores, al ser costoso el despido, tienen poco incentivo para las nuevas contrataciones.

Muy en sintonía con la postura que anteriormente mencionamos como "ilusión óptica" con respecto al desempleo juvenil, hay algunas corrientes que relativizan la gravedad del problema. En primer lugar, habría más brechas entre la formación y las habilidades requeridas en el mercado, en el caso de los adultos que en los jóvenes, por lo que los esfuerzos en capacitación deberían orientarse a aquellos más que a estos últimos. En el mismo sentido, los jóvenes pueden ir incorporando las habilidades y conocimientos requeridos a lo largo del tiempo y, por ende, las dificultades iniciales de inserción tenderían a mejorar al incorporar más experiencia a la vida laboral. "Además, se argumenta que la evaluación de la gravedad 
de los problemas de la inserción laboral juvenil requiere diferenciar entre los y las jóvenes que son jefes(as) de hogar de aquellos(as) que no lo son. Por una parte, altas tasas de desempleo para jóvenes que no son jefes(as) de hogar pueden reflejar una mayor posibilidad para buscar un empleo que mejor satisfaga sus expectativas. Dado que tienen un conocimiento limitado del mercado de trabajo y de los puestos realmente disponibles, esta búsqueda demoraría más que en el caso de los adultos. Como, además, viven en un hogar donde no son la principal fuente de ingreso, la presión de emplearse es menor. En consecuencia, su tasa de desempleo es típicamente más alta que aquella de los adultos. En contraste, los y las jóvenes que sí son jefes(as) de hogar generalmente no pueden quedarse sin ingresos por un tiempo extendido, por lo que sería más que todo la inserción de estos jóvenes que indicaría la gravedad de los problemas de desempleo juvenil" (Weller 2003: 18).

Otro elemento que habría que tener en cuenta es el hecho de que los jóvenes han aumentado el nivel educacional de sus padres, por lo cual sus expectativas laborales muchas veces superan con creces lo que el mercado les ofrece; de esta manera, tienden a menospreciar los empleos de obreros y operarios y a preferir los de oficinistas e incluso los trabajos por cuenta propia, que les dan la promesa de tiempos personales más flexibles y mejores salarios que los empleos del sector formal (Schkolnik 2005: 15).

\section{Propuestas de políticas para el empleo juvenil}

Sin duda que las políticas de fomento del empleo juvenil están estrechamente relacionadas con las hipótesis que se generan para explicarlo, ya sean las que hemos esbozado en el punto anterior u otras.

La más general de las políticas, que se corresponde directamente con la primera hipótesis que vincula las causas del desempleo juvenil con factores generales del funcionamiento de la economía, tiene que ver con una política de empleo ${ }^{3}$ que oriente la política macroeconómica hacia un crecimiento económico alto, sostenido y

3 Se entiende por políticas de empleo a los "instrumentos macroeconómicos y sectoriales que influyen en la magnitud y la composición del producto y que, por consiguiente, influyen en la magnitud y composición del empleo y las remuneraciones absolutas y relativas"(Weller 2005: 23) 
sustentable, con altas tasas de inversión. A este respecto, hay que señalar la conciencia creciente respecto de la necesidad de crear un entorno propicio para la generación de empleo, lo que pasa no sólo por una cuestión de índole cuantitativa, sino de enfocarse desde la óptica del desarrollo y de la inclusión, en donde la existencia de jóvenes trabajando no sólo es deseable desde la perspectiva de la eficiencia, sino también de la justicia social (Weller 2005: 37).

Dimos cuenta anteriormente también de las hipótesis que apuntan a una flexibilización del mercado laboral. Efectivamente, en muchos países de la OCDE se ha procedido a ajustar las políticas de contratación para hacer más viable la entrada de los jóvenes al mercado de trabajo; pese a ello, aunque se trata de una medida que aumenta las probabilidades para acceder posteriormente a un empleo estable, también puede aumentar la cantidad de contrataciones a corto plazo que se combinan con periodos de desempleo, en ciclos que incluso pueden durar toda la vida de un trabajador. Por otra parte, tampoco hay certeza de que una modificación en la legislación laboral o en la institucionalidad ligada al trabajo produzca los efectos deseados, toda vez que la mayoría de los jóvenes se inserta en la economía informal (Ministerio del Trabajo y Previsión Social, Gobierno de Chile; 2005: 17) .

Algunas políticas más específicas podrían agruparse en torno a cuatro ejes:

- Aquellas que apoyen la inserción al primer empleo

- Las que favorezcan la empleabilidad juvenil

- Las que potencian el espíritu emprendedor de los jóvenes

- Políticas para grupos específicos

\section{Políticas que apoyen la inserción al primer empleo}

En cuanto a estas políticas, como se puede apreciar en la mayor parte de las hipótesis, "la primera inserción suele ser uno de los temas

4 Se señala en ese mismo documento: "Por ejemplo, la flexibilización introducida en los ' 90 en Argentina, Colombia, El Salvador y Perú no permitió crear el número de empleos previsto, pero incrementó el número de trabajadores sin contrato o con contratos de plazo fijo" (Ministerio del Trabajo y Previsión Social, Gobierno de Chile 2005: 17) 
más complejos con respecto al desempleo juvenil, sobre todo en términos de los requerimientos de buena educación y experiencia que resultan ser los factores claves para marcar la diferencia. Para efectos de este aspecto, entonces, es vital tomar medidas con respecto a los contactos entre la demanda y la oferta laboral. Como ejemplos podemos ver: charlas de empresarios y servicios locales de empleo sobre opciones laborales en el entorno local, sumadas a visitas a empresas; charlas de adultos jóvenes en colegios sobre experiencias de inserción laboral; pasantías y prácticas, trabajos de temporada y de tiempo parcial; programas de apoyo a la inserción que impliquen el desarrollo de habilidades sociales aptas para mejorar la empleabilidad; apoyo al microemprendimiento juvenil" (Weller 2003: 67).

Un marco para estas políticas lo ha constituido el Pacto Europeo por la Juventud, que se ha comprometido con un enfoque integrado donde se entremezclan la educación, la formación, el aprendizaje permanente y la integración social. La idea ha sido crear empleo y mejorar la transición entre la escuela y el trabajo, mejorar las condiciones laborales y la empleabilidad.

En la misma línea, en algunos países se ha alargado la educación obligatoria, pero incluyendo hacia el final del ciclo la formación en el lugar de trabajo. "El desarrollo de calificaciones esenciales para el trabajo forma parte de la reforma de la educación y formación profesional en el mundo, las que incluyen nuevas habilidades transversales tales como comunicación, relaciones interpersonales, capacidad para aprender y resolver problemas, conocimiento de las Tecnologías de la Información y Comunicaciones. Tales habilidades son transferibles entre ocupaciones, empresas y sectores, siendo esenciales para la empleabilidad y el aprendizaje permanente" (Ministerio del Trabajo y Previsión Social, Gobierno de Chile 2005: 17).

En los países en que la problemática de la primera inserción se complejiza con la existencia de una alta rotación en los primeros años de vida laboral, las políticas no pueden abocarse exclusivamente a la primera inserción, sino que deben apoyar también la reinserción. En este sentido, podemos tener en cuenta medidas como las reformas a los contratos de formación, obtención de credenciales laborales y sistemas de información laboral.

Las reformas a los contratos de formación tienen que ver con el perfeccionamiento de la regulación laboral, de forma tal de evitar que 
la capacitación, en vez de formar jóvenes, en realidad termine sólo por abaratar los costos de la mano de obra. Para ello es fundamental que los contratos definan con precisión lo que se entiende por "formación", que se regule con precisión con respecto a los contratos de prácticas, que se mejoren los sistemas de supervisión y que las empresas estén obligadas a certificar la formación que entreguen a los jóvenes. Todas estas medidas requieren, por consecuencia, de un buen sistema de acreditación que haga cumplir los contratos estipulados.

Con respecto a las credenciales laborales, es necesario impulsar medidas en la dirección de crear sistemas integrados que permitan a los jóvenes acceder a los documentos que solicitan los empleadores a precios razonables y en tiempos no excesivos. En pocas palabras, la documentación que se exige a los jóvenes debería ser accesible para todos los sectores con un desembolso mínimo de dinero y sin discriminación.

Los sistemas de información laboral, por su parte, deben cumplir con las siguientes condiciones: debe ser un sistema que coordine a las distintas instancias ministeriales, entregando servicios completos y no reducidos a la simple colocación de personas en puestos de trabajo; debe servir para la acreditación de jóvenes de escasos recursos en el mercado laboral, ayudando a perfeccionar la información para un sector con más dificultades de acceso al mercado de trabajo; y debe regular estrictamente el funcionamiento de los sistemas privados y públicos de colocación laboral (Chacaltana; 2006: 194-202).

\section{Políticas que favorezcan la empleabilidad juvenil}

En lo relativo a estas políticas, básicamente en términos de educación, formación para el trabajo y experiencia.

En el caso de la educación, tiene que haber políticas fuertes para disminuir las tasas de deserción escolar, para mejorar la calidad de la educación y para articular la educación con el trabajo. La evidencia empírica muestra que la mayor parte de los jóvenes no se insertan de inmediato en el mercado laboral luego de haber terminado su formación (incluso los que tienen más años de estudio), lo que puede ser un incentivo a la generación de alternativas de formación postsecun- 
daria de bajo costo, pero funcionales a los futuros empleos. "Por otro lado, es necesario redoblar esfuerzos para que la educación pueda proveer a los jóvenes de dos elementos que son cruciales en el mercado de trabajo: la capacidad de aprender leyendo (entender lo que leen) y la capacidad de aprender haciendo (aplicar el conocimiento a casos concretos). Es cierto que éste es un trabajo de largo plazo, pues el sistema educativo tiene numerosos problemas, pero se trata de una precondición indispensable en la solución de los problemas laborales de los jóvenes" (Chacaltana 2006: 205).

Por otra parte, la capacitación y la experiencia se transforman en dos herramientas estratégicas fundamentales para disminuir el desempleo juvenil, pues pueden generar en su conjunto herramientas para promover la movilidad ascendente de los jóvenes. La experiencia debe ser fortalecida y reconocida a través de sistemas de certificación serios y de calidad; en el caso de la capacitación, debe existir un mercado apropiado, eficiente y rigurosamente fiscalizado. Por el lado de la capacitación, debe haber apoyo a la demanda con rigurosidad de contenidos y, como se hace hoy en muchos países, a través de un sistema de formación por competencias. Por el lado de la oferta, es válido examinar los resultados y enseñanzas de los Programas ProJoven impulsados en varios países de América: los mayores retornos de la capacitación se producen cuando ésta se lleva a cabo en la propia empresa, cuando los aprendices aprueban los módulos teóricos y los prácticos y cuando las empresas están activamente involucradas en el proceso 5 .

\section{Políticas que potencian el espíritu emprendedor de los jóvenes}

En lo relativo a las políticas que potencian el espíritu emprendedor de los jóvenes. La promoción del emprendimiento es una orientación de política que debe comenzar en la escuela y debe involucrar no sólo al sector público, sino también al privado, con especial énfasis en los medios de comunicación de masas.

5 "Esto no es una sorpresa. La capacitación debe ser pertinente para poder ser útil, y esta pertinencia sólo se asegura cuando la empresa se involucra de algún modo. Por esta razón, es claro que cualquier estrategia de capacitación que se promueva en el ámbito nacional debe otorgar un papel principal a las empresas" (Chacaltana 2006: 206). 
Debe haber programas que premien también las acciones donde se tomen riesgos. Como señala Juan Chacaltana, en algunos países “... se han lanzado programas que premian la iniciación de un segundo negocio (después del primer fracaso), como señal de que la sociedad premia este tipo de esfuerzos. La racionalidad de este tipo de intervención es similar al 'premio' que se da en programas de empleo asalariado a aquellos jóvenes que se acercan a solicitar empleo a una bolsa de empleo o a un programa de formación: tienen variables no observables favorables a la empleabilidad y, por tanto, es conveniente invertir en ellos" (Chacaltana 2006: 204). Lo anterior debería ir acompañado por facilidades para los negocios y reducción de costos (en tiempo y dinero) para los trámites para generar empresas y premios a la innovación, entre otros.

\section{Políticas para grupos específicos}

Políticas para grupos específicos, con especial énfasis en las mujeres jóvenes, los discapacitados, los desertores del sistema escolar y los jovenes más pobres. Estos programas se orientan no sólo por las conveniencias de tipo económico que genera en un país la existencia de menores índices de desempleo (mayor eficiencia en el uso de los recursos, menor propensión a la marginalidad y el descontento social, mayor retorno de las inversiones en capacitación, etc.), sino que se fundamentan en la construcción de una sociedad más justa, que permite condiciones más igualitarias de vida, apoyando a los que se encuentran en desventaja relativa con respecto a otros actores sociales.

Como señala Chacaltana, el mercado de trabajo es más complejo que otros mercados porque es el único en donde los bienes transados son servicios que los proporcionan personas y estas mismas participan en el proceso de formación. "Esto tienen su lado positivo y su lado negativo. En el lado positivo, aquellas personas que más sepan transmitir a los compradores las características de su trabajo tendrán más posibilidades de éxito en el mercado laboral. Sin embargo, existen personas que no tienen esas habilidades, y se hacen necesarias políticas de apoyo a la inserción y reinserción, con el propósito de facilitar este proceso. En el lado negativo, esta propiedad de la mano de obra es una de las causas por las que existe exclusión laboral. En realidad, esto es lo que determina que se terminen conformando grupos dentro del mercado de trabajo, y determina 
también que las relaciones personales formen el mecanismo más importante para acceder a un puesto de trabajo. El punto es que en el mercado de trabajo tienden a formarse grupos, los cuales se conforman en función de la raza, la apariencia, el sexo, la educación, el barrio, etc. Y el problema es que existe un patrón de contratación que discrimina a algunos de estos grupos de acuerdo con determinados atributos, lo cual genera una clara desventaja para ciertos trabajadores. Romper estos mecanismos de discriminación es ciertamente difícil y los jóvenes, en especial aquellos excluidos, enfrentan las consecuencias cada día" (Chacaltana 2006: 211).

Un caso especial lo constituyen las mujeres jóvenes desempleadas, especialmente las madres solteras. En esta situación, es deseable la promoción de políticas que se dirijan a apoyar la incorporación de estas jóvenes al mercado laboral y a su mantención en él, con medidas como guarderías de niños u otros sistemas de cuidado infantil.

\section{Algunas experiencias internacionales exitosas y el caso de Chile}

En el marco de políticas generales de empleo, destaca la experiencia de dos países: Eslovenia y Costa Rica. En el primer caso, el tema se abordó en el paso de Eslovenia a una economía de mercado que se caracterizó principalmente por la gradualidad y la combinación de reformas económicas y control del equilibrio fiscal, la balanza de pagos y la inflación. El gobierno creó, así, un entorno favorable para la inversión interna y extranjera, lo que favoreció la creación de empleos. El servicio público de empleo, además, generó políticas de mediación laboral y otras políticas activas de mercado de trabajo, que incluía servicios de orientación profesional para los jóvenes que buscan trabajo por primera vez, promoción de pequeñas empresas, pasantías e iniciativas locales para los desempleados. Hay que destacar también que el Consejo Económico y Social de Eslovenia fomentó la estabilidad laboral y la interlocución tripartita para construir acuerdos laborales que combinaran aumentos salariales y mantención de equilibrios macroeconómicos. Por último, existió un apoyo decidido a la creación de empresas, las que se convirtieron en las principales generadoras de empleo.

El caso de Costa Rica también se basó en el crecimiento económico sostenido del país entre los años 1990 y 2003. La estabilidad 
económica estuvo complementada con políticas destinadas a atraer la inversión extranjera en actividades exportadoras, que al utilizar tecnología avanzada requieren trabajadores bien calificados. Por otro lado, se fortaleció la política de educación en todos los niveles, se mejoró la política de formación y se mejoró el sistema de capacitación orientado a los sectores más pobres de la población. Los recortes al empleo que generaba el sector público fueron compensados con la promoción de las inversiones en agricultura, industria y turismo.

Pese a estos casos, la experiencia tiende a mostrar que las políticas generales de crecimiento económico no bastan por sí mismas para generar empleo, sino que ellas deben combinarse integral y efectivamente con las políticas sociales y específicamente con las políticas educativas.

Algunos países han enfatizado en políticas sectoriales exitosas, tratando de sacar provecho de ventajas competitivas. Un caso ha sido el de la agricultura, donde países como China y Tailandia han decidido intervenir intensivamente, o Ecuador y Colombia en la floricultura. En todos estos casos, la ampliación del trabajo ha traído como consecuencia la disminución del desempleo juvenil.

Otro sector que ha traído dividendos ha sido el de los servicios de hotelería, restauración y turismo, que han brindado abundante empleo para trabajadores con escasa o nula capacitación profesional. Esto ha ocurrido en países como Croacia o Sudáfrica.

En países con un fuerte desarrollo de las Tecnologías de la Información y la Comunicación, como es el caso de Hong Kong, Corea, India y Malasia, se ha generado mucho empleo para jóvenes con distintos niveles educativos.

En Reino Unido, Albania y Lituania se ha desarrollado el sector de servicios deportivos; en Irlanda y Canadá las artes y la cultura y en China e India el sector salud.

En el ámbito del mejoramiento de la empleabilidad de los jóvenes destacan las siguientes iniciativas por países (OIT 2005: 54 y Ss.):

- $\quad$ En Reino Unido, el Programa New Deal for Young People ofrece una serie de servicios para jóvenes entre 18 y 24 años y desempleados desde hace por lo menos seis meses; los servicios incluyen: 
búsqueda de empleo, orientación profesional y formación de diverso tipo. Las colocaciones están subvencionadas y se realizan actividades de seguimiento.

- En Canadá se creó la Estrategia de Empleo para los Jóvenes, que trata de que éstos accedan a la información, desarrollen competencias y adquieran experiencia profesional para incorporarse con éxito al mundo del trabajo. El Programa coordina distintas instancias gubernamentales las que, a su vez, tienen redes con organizaciones de la comunidad y diversas instancias empresariales.

- En Japón existe un programa que abarca a cuatro ministerios, llamado el Young Peoples's Self-Support and Challenge Plan y que tiene por objetivo el promover la autosuficiencia y proporcionar empleo a todos los jóvenes motivados.

- En Francia, el Programa Nuevos Servicios, Nuevos Empleos, ofrecía subvenciones salariales de hasta el $80 \%$ a los empleadores que contrataran a trabajadores jóvenes. Se creó un sistema de supervisión y se promovió la creación de nuevos trabajos hasta el momento no cubiertos por las empresas, como la asistencia personal, la seguridad, el acceso a la cultura, apoyo educativo y educación ambiental.

Habría que destacar también en un lugar especial las políticas referidas al mejoramiento de los servicios de información para el mercado de trabajo. A este respecto tenemos el caso, por ejemplo, de Reino Unido, que creó el Connexxions Direct, un servicio de apoyo para jóvenes de entre 13 y 19 años, destinado a proporcionarles información y orientación sobre las oportunidades disponibles, además de hacer seguimiento y crear una red de consejeros personales para prestar asesoría y acompañamiento.

El Ministerio de Desarrollo de los Recursos Humanos de Canadá creó el Job Futures, una herramienta para proporcionar información sobre el mercado de trabajo, enfatizando en la relación entre éste y los logros educativos. Paralelamente, el Job Bank es un listado electrónico de empleos y oportunidades empresariales que ofrecen empresarios de todo el país; la información se puede obtener desde sitios públicos con acceso libre a internet que se ubican en centros de recursos humanos, bibliotecas públicas y centros comunitarios. 
Con respecto a la experiencia chilena, desde los años ' 90 se han implementado una serie de políticas como la reforma educacional, la reforma al Código del Trabajo y una serie de programas específicos para la inserción laboral juvenil. Estos últimos han estado centrados en la convicción de la importancia del capital humano para el desarrollo del país y en la necesidad de generar condiciones más igualitarias de acceso a la educación y el trabajo para jóvenes de distintos estratos socioeconómicos.

Destaca especialmente el Programa Chile Joven, orientado por cuatro líneas:

- Una línea de Capacitación y Formación para el trabajo, habilitando a jóvenes para su inserción social y laboral y orientada a cubrir nichos de formación en un nivel de semicalificación

- Aprendizaje Alternado, combinando el trabajo en aula con un entrenamiento en una empresa por un periodo de entre 3 a 12 meses

- Habilitación para la creación de microempresas, con capacitación en gestión y asistencia técnica

- Capacitación con experiencia laboral en empresas (Fernández y Charlin; 2006: 188)

El Programa Chile Joven se orientó a jóvenes de bajos ingresos que se encontraban fuera del sistema escolar y que estaban desocupados y con deseos de trabajar. En el marco de una orientación general de política de generar "igualdad de oportunidades", se trataba de brindar condiciones de equidad para el acceso al mercado de trabajo de los grupos de jóvenes más desfavorecidos.

La primera fase del Programa se ejecutó entre los años 1991 y 1995 y se alcanzó a cubrir a 120.000 beneficiarios. Desde 1996 se dio inicio a una segunda etapa, introduciéndose las siguientes modificaciones: cambian sustantivamente los sistemas de incentivos a los ejecutores, estableciéndose una modalidad de pago relacionada directamente con los resultados de la inserción social; se establece también una fase de seguimiento de los egresados de su práctica profesional. Se incrementa la importancia relativa de la modalidad de Aprendizaje Alternado, con una prioridad en la focalización territorial. Se fortalece la coordinación interministerial para la ejecución 
y evaluación del programa; se cambian los énfasis desde la inserción social a la inserción laboral. Por último, se abre una línea de trabajo especialmente orientada a la vinculación entre demanda laboral y el personal semicalificado.

Entre los principales resultados de la evaluación del Programa se contaron:

- Las dificultades para reclutar a los grupos más vulnerables, pese a lo cual hubo una alta participación femenina. "Esto podría deberse, por una parte, a que la deserción femenina es menor, por lo tanto la matrícula femenina beneficiaba los subsidios pagados, por cada joven que se mantenía en el programa, a los organismos capacitadotes, incentivando a éstos para el reclutamiento femenino. Por otra parte, la mayor participación femenina probablemente estuvo vinculada a la creciente demanda de las mujeres jóvenes por insertarse en el mercado laboral y desarrollar un proyecto personal autónomo" (Fernández y Charlin 2006: 190).

- Existió baja cobertura del Programa en regiones, por lo que los beneficiarios se concentraron mayoritariamente en sólo tres regiones.

- Hubo un positivo impacto del Chile Joven en el nivel ocupacional de los beneficiarios, aunque se constató una relación entre los saldos positivos y los niveles educacionales de los jóvenes. El que mostró mejores resultados fue el de Aprendizaje Alternado y el que presentó mayor inscripción femenina el de microemprendimiento, toda vez que este tipo de trabajos permite la posibilidad de compatibilizar el trabajo con los quehaceres del hogar y el cuidado de los hijos.

- Se logran mejores resultados cuando existe una vinculación entre los aprendizajes y las experiencias en las empresas.

En el año 2002 se creó otro programa, el Chile Califica, que representa un salto cualitativo en términos de políticas de empleo en Chile, en tanto tiene un carácter multisectorial (su ejecución depende los ministerios de Educación y del Trabajo y de la Dirección de Presupuestos del Ministerio de Hacienda) y pretende articular de mejor modo el mundo de la educación con el del empleo remunerado. "Este programa contempla el desarrollo de tres componentes, 
con el propósito de: 1) atender los requerimientos de formación de competencias para el trabajo, mediante alfabetización, mayor escolaridad y capacitación; 2) mejorar la formación técnica; y 3) instalar un marco nacional de competencias laborales e itinerarios de formación técnica y un sistema de información para la educación y capacitación permanente" (Fernández y Charlín 2006: 192).

El primer componente trata de ampliar las oportunidades de nivelación de estudios básicos y medios y capacitación para el trabajo a través de una modalidad de estudio flexible, con un sistema de evaluación y certificación de los mismos. El segundo componente apoya la formación técnico-profesional, incentivando el mejoramiento de la calidad y la actualización de los equipos docentes. El tercer componente trata de generar un sistema articulado de educación y capacitación permanentes, creando un sistema nacional de competencias e itinerarios de formación técnica.

\section{Reflexiones Finales}

Como se ha señalado abundantemente en este trabajo, el desempleo es uno de los problemas más relevantes y preocupantes de las sociedades contemporáneas y no afecta sólo a los países subdesarrollados, aunque es probable que en ellos lo haga con mayor crudeza. Pese a ello, la cesantía no es el único problema que enfrentan los jóvenes que se incorporan al mercado del trabajo, por lo que es necesario subrayar que mostrar la situación no puede conducirnos a obviar otros temas no menos dramáticos.

En efecto, la evidencia empírica internacional muestra que muchos jóvenes en todo el mundo trabajan un número inaceptable de horas, de manera informal, intermitente e insegura (OIT 2005: 14 y ss.). En muchos países subdesarrollados los desempleados son abrumadoramente jóvenes y también lo son quienes se desempeñan en el sector informal tanto urbano como rural. Por otra parte, en muchas de estas economías existen políticas enfocadas a la problemática del desempleo juvenil, pero muy pocas de ellas se atienen a la de las malas condiciones de trabajo de los jóvenes. Para ciertas posturas teóricas y políticas, la dicotomía empleo-desempleo habría perdido, entonces, su significado, en circunstancias en las que estar empleado podría en realidad ser sinónimo de ausencia de servicios de seguridad social, provisionalidad extrema del trabajo o carencia de 
autoestima para negociar mejores sueldos o condiciones laborales. En este caso, cuando un joven trabaja en un empleo que le exige por debajo de sus capacidades, hablamos de desaprovechamiento del capital humano de un país.

En un sentido parecido, algunas cifras sobre desempleo juvenil pueden ser, en realidad, un tanto engañosas, pues ante un mercado de trabajo especialmente adverso o precario, muchos jóvenes (especialmente mujeres), pueden ni siquiera intentar la búsqueda de trabajo, por lo que no aparecen en los datos oficiales. Algunos de ellos estarían derechamente "haciendo nada", sin educación y soportando malas condiciones de vida en sus hogares o fuera de ellos.

En razón de estos elementos, resulta fundamental hacerse cargo de algunos desafíos para las políticas de desempleo juvenil.

En primer lugar, hay un reto a la integralidad que pasa por desbordar los límites del desempleo y acercarse al tema general de la precariedad de la condición del asalariado joven, con propuestas que no sólo se aboquen a la oferta, sino que corrijan también los defectos e irregularidades de la demanda de empleo juvenil.

En segundo lugar, y si tal como lo hemos esbozado, existe una segmentación del mercado de trabajo de los jóvenes, debiesen existir también políticas laborales para grupos específicos, atendiendo especialmente a los jóvenes discapacitados, mujeres, jefes de hogar, entre otros.

Siguiendo de cerca los planteamientos de (Schkolnik 2005: 45-46), la política no puede hacerse cargo exclusivamente de la situación actual de los jóvenes, sino que debe tener alguna capacidad de avizorar cuál es el panorama social en el mediano plazo, para prevenir y resolver problemas venideros. En ese sentido, debería tener en cuenta a lo menos tres tendencias: el paso desde un aprendizaje por experiencia (de acuerdo al cual los jóvenes adquirían un oficio en el seno de su familia y continuaban la tradición laboral de sus padres) a un aprendizaje permanente de habilidades que sólo entrega el sistema escolar y que hacen más urgente hoy la existencia de políticas anti-deserción y la inclinación de los sistemas educativos hacia la formación en destrezas abstractas y sociales por sobre las habilidades manuales. 
En la "era del fin del trabajo", los jóvenes enfrentarán de modo creciente un mundo con menos empleos, con trabajos más cortos, más flexibles y menos estables. En una sociedad de este tipo se requiere de una mentalidad de formarse permanentemente, y un diseño de políticas sociales que contribuyan a desarrollar la capacidad de aprender a aprender, en el fortalecimiento de las redes laborales y en la creación de un sistema integrado de capacitación con una oferta variada y ascendente.

Por último, de modo creciente la inserción laboral ya no es un proceso natural en la vida de las personas ni está dada la articulación entre la educación y el trabajo. Para enfrentar eso se requiere de políticas que promuevan la autogeneración de empleos y de protección social para periodos de cesantía, con una oferta de formación que aproveche los tiempos de desocupación.

Todo esto, como lo hemos dicho anteriormente, no sólo es con la finalidad de rentabilizar el capital humano; es, por sobre todo, una cuestión de equidad y de construcción de una sociedad en la que cada joven tenga la oportunidad de dignificar su vida a través del trabajo e integrarse al proceso de construir su propia felicidad desde el desarrollo pleno de sus capacidades.

\section{Referencias Bibliográficas}

CHACAlTANA, J. (2006). Políticas de empleo para jóvenes. Perú: CEDEP, CEPAL, GTZ.

CHILE, MINISTERIO DEL TRABAJO Y PREVISIÓN SOCIAL (2005). Promoción del Empleo Juvenil. Programas públicos y cambios normativos. Observatorio Laboral No 18.

FERNÁNDEZ, P. y CHARLIN, M. (2006). Políticas y programas de apoyo a la inserción laboral de los y las jóvenes en Chile. En: Charlin de Groote, M., Weller, J. (eds.) Juventud $y$ mercado laboral: brechas y barreras. Santiago de Chile: FLACSO / CEPAL.

GALLART, M. (2003). La formación para el trabajo y los jóvenes en América Latina. Santiago de Chile: CEPAL.

GARCÍA, J., GUTIÉRREZ, R. (1996). Inserción laboral y desigualdad en el mercado de trabajo: cuestiones teóricas. Revista REIS, 75.

OFICINA INTERNACIONAL DEL TRABAJO (2005). El empleo de los jóvenes: vías 
para acceder a un trabajo decente. Conferencia Internacional del Trabajo 93. Ginebra: AUTOR.

(2006). Tendencias Mundiales del Empleo Juvenil. OIT, Ginebra

MARTíNEZ, E. (1997). Desempleo juvenil en Chile: ¿discriminación o ilusión óptica?. Boletín CINTERFOR № 139-140

SCHKOLNIK, M. (2005). Caracterización de la inserción laboral de los jóvenes. Serie Políticas Sociales N ${ }^{\circ} 104$. Santiago de Chile: CEPAL.

WELLER, J. (2003). L L L problemática inserción laboral de los y las jóvenes. Serie Macroeconomía del Desarrollo, № 28. Santiago de Chile: CEPAL

(2005). Problemas de empleo, tendencias subregionales y políticas para mejorar la inserción laboral. Serie Macroeconomía del Desarrollo No 40 . Santiago de Chile: CEPAL. 